\title{
TOWARDS A DATA LINK FOR UNMANNED AVIATION: DLR FLIGHT MEASUREMENT CAMPAIGN FOR C2 DATA LINK DEVELOPMENT
}

\author{
Daniel M. Mielke, German Aerospace Center DLR, Wessling \\ Nicolas Schneckenburger, German Aerospace Center DLR, Wessling
}

\begin{abstract}
Unmanned Aircraft (UA) are expected to play a more and more important role in global aviation during the next years. Besides the "smart" onboard control equipment leading to a certain amount of autonomy of the vehicle, a reliable data link ("Command and Control", C2-link) is a key enabler to control UA in civil airspace. This $\mathrm{C} 2$ link is used to exchange information between the UA and the remote pilot, for example positioning information, telemetry data or flight trajectories, respectively. None of the currently available aeronautical communication standards can fulfil the requirements for such a multi-user, multi-point datalink in terms of reliability, flexibility, data integrity, robustness and latency.

The German Aerospace Center (DLR) initiated the "C-Band Digital Aeronautical Communication System" (CDACS) project to develop a C-band data link for this purpose. Part of this project is a flight measurement campaign to evaluate initial CDACS waveform designs, besides performing radio channel measurements of the terrestrial ground-air channel in C-band. Detailed knowledge on the behavior of the physical communication channel is crucial for an optimal design of a new communication system. Therefore, it is necessary to perform C-band channel measurements (aka "channel sounding") and use the collected information to develop a channel model. In turn, this channel model can be used to optimize and evaluate the CDACS waveform design in simulations. The bandwidth used for the C-band channel measurements of this campaign was $\sim 50 \mathrm{MHz}$ whereas the CDACS waveforms tested spanned a bandwidth of $\sim 1.5 \mathrm{MHz}$. Multiple flight routes and maneuvers are chosen to collect information on different flight scenarios, e.g. takeoff and landing, hard banking and en-route.
\end{abstract}

\section{Introduction}

Unmanned aviation is gaining interest both in industry and science for the last years. Unmanned aircraft (UA) have been shown to be applicable to various tasks in the fields of transportation/logistics, healthcare, agriculture monitoring/surveillance, and military/defense.

Although UA will reach a comparatively high level of autonomy, a communication link between a remote pilot and the vehicle is still indispensable. This Command and Control (C2) link is used only for data immediately required to operate the UA and not for any additional traffic, called payload data. Examples for $\mathrm{C} 2$ data are

- flight trajectories, way points, and other technical control commands transmitted from the remote pilot to the UA, called forward link (FL)

- positioning, status and weather information as well as a video link ${ }^{1}$ transmitted from the UA to the remote pilot (RP), called reverse link (RL).

The approach of the German Aerospace Center (DLR) to such a $\mathrm{C} 2$ link for UA in non-VLL (non very low level) airspace is called C-Band Digital Aeronautical Communications System (CDACS). CDACS is designed to fulfil the requirements in terms of throughput, latency, availability, and continuity. First concepts for the terrestrial CDACS have been published in [1] and [2].

To design a wireless communication system like CDACS, knowledge on the conditions of the communications channel, in this case the airground/ground-air channel $(\mathrm{AG})^{2}$ is essential. This knowledge can be exploited in two ways: First, the physical properties have a direct influence on the waveform parameters of the system. Second, the

\footnotetext{
1 The demand for a video link is still discussed in the unmanned aviation community.

${ }^{2}$ The authors assume a reciprocal channel behavior.
} 
gained channel information is used to build a channel model which can be applied to evaluate the communications system's performance. The channel information is gained by a process called channel sounding. In channel sounding a known signal is transmitted through the channel. The signal is recorded and analyzed at the receiver side. By comparing the transmitted and the received signal we gain information on the channel characteristics.

In the following paper we describe the flight trials conducted to investigate the AG channel. The setup comprises a ground based transmitter and an airborne receiver located on a jet aircraft. Additionally to the task of channel sounding, the setup is exploited to investigate the performance of the current version of the CDACS waveform. Thus, the current CDACS design concepts may be evaluated.

The remaining paper is structured as follows: We start by explaining the transmitted waveforms. The following two sections are dedicated to the hardware setup and the flight routes during the campaign, respectively. The paper ends with some example results and a conclusion and outlook section.

\section{Transmitted Waveforms}

The proposed frequency band for CDACS is $5,030 \mathrm{MHz}-5,091 \mathrm{MHz}^{3}$, i.e., $61 \mathrm{MHz}$ of bandwidth. However, the center frequency used in the flight campaign is $5,200 \mathrm{MHz}$ due to hardware availability. The authors do not expect a significant difference in the physical behavior of the radio channel considering the comparatively small frequency delta of $\sim 100 \mathrm{MHz}$. The available hardware limited the theoretical bandwidth to $50 \mathrm{MHz}$.

Generally speaking, two types of signals were used in the campaign; we provide a detailed description of both signals in the following.

\section{Channel Sounding}

For channel sounding we employ a multi-tone signal similar to OFDM. The channel sounding

\footnotetext{
${ }^{3}$ This band is assigned to the Microwave Landing System (MLS) at the moment. Since it is not widely used, a reassignment to $\mathrm{C} 2$ links for UA is discussed, see [?].
}

signal is a pre-generated vector $\boldsymbol{s}_{\mathrm{CS}}$ of complex samples that is replayed on an arbitrary waveform generator (AWG) in an infinite loop. Assuming a vector length of $N$, the $n$-th item of the vector in frequency domain is given by

$$
X_{n}=e^{j \pi \frac{(n-1)^{2}}{N}}, \forall n \in\left\{-\frac{N}{2}+N_{G}+1, \ldots, \frac{N}{2}-N_{G} \mid n \neq 0\right\},
$$

where $N_{G}$ denotes the amount of guard carriers. The quadratic phases of $X_{n}$ are called Newman Phases [3] and are known to have a beneficial effect on the resulting signal's peak to average power ratio (PAPR).

The time domain signal is generated by an Inverse Fast Fourier Transform (IFFT) of length $N$ :

$$
\boldsymbol{s}_{\mathrm{CS}, 0}=\operatorname{IFFT}_{N}\left\{\left[0,0, \ldots, X_{n-1}, X_{n}, X_{n+1}, \ldots, 0,0\right]\right\} .
$$

The IFFT-operation at the end of the signal generation step can be interpreted as a modulation, where each item of the frequency domain vector gets modulated on a different subcarrier of the resulting time-domain signal. This corresponds to the modulation process of OFDM. While in OFDM the phases are typically chosen from a modulation alphabet (and are therefore discrete), the chosen Newman phases effectively reduce the signals PAPR compared to a standard OFDM signal.

To avoid signal distortions by the amplifier, the peak power of a transmitted signal should never exceed the upper bound of the linear domain of the amplifier. Thus, the operating point must be chosen to fulfil this condition, i.e., the whole signal power gets reduced by an appropriate PAPR-back-off. This makes the amplifier operating in a suboptimal operating point most of the time. The probability of a high peak of an OFDM signal's power, thus resulting in a high PAPR, increases with the number of subcarriers used, since it becomes more likely that a considerable amount of subcarriers is in-phase and adds up to a high peak.

Nevertheless, OFDM is a popular modulation system and the minimization of the PAPR is subject to a lot of ongoing research. One efficient approach to mitigate the PAPR problem is to iteratively clip and filter the desired signal, until a certain stopping criterion is fulfilled e.g. a target PAPR value or a maximum number of iterations $K$ has been performed. This strategy implies two disadvantages 
that limit its application in many cases: First, the clipping process adds distortions to the signal that may degrade the system's performance. Second, the iterative processing adds a significant processing delay. However, both the distortion and the processing delay are not an issue for channel sounding, what makes this approach very attractive for this application.

The iterative process is defined as

$$
\boldsymbol{s}_{\mathrm{CS}, k}=\operatorname{Filt}\left\{\operatorname{Clip}_{a}\left\{\boldsymbol{s}_{\mathrm{CS}, k-1}\right\}\right\}, \forall 0<k \leq K,
$$

where $a$ is the clip threshold of the clipping process and a rectangular low-pass filter is used for the filtering process. The last step of the generation is given by $\boldsymbol{s}_{\mathrm{CS}}=\boldsymbol{s}_{\mathrm{CS}, K}$.

The parameters used for generating the channel sounding signal used in the campaign are $N=2048, N_{G}=5, a=0.95$ and $K=80000$. The signal is upsampled by a factor of $f_{\text {up,CS }}=2$ and played on the AWG with a sample rate of $100 \mathrm{MSample/s}$. Considering the guard carriers, the effective bandwidth of the transmitted signal is 49.78MHz. The guard carriers were chosen to circumvent problems caused by frequency shifts implied by oscillator inaccuracies and Doppler frequency.

Table 1. CDACS OFDM Configurations

\begin{tabular}{|l|c|c|c|c|}
\hline Property\Conf.ID & $\mathbf{1}$ & $\mathbf{2}$ & $\mathbf{3}$ & $\mathbf{4}$ \\
\hline FFT Length & 128 & 128 & 64 & 64 \\
\hline $\begin{array}{l}\text { Sub-carrier } \\
\text { Spacing }[\mathrm{kHz}]\end{array}$ & 11.34 & 11.34 & 22.68 & 22.68 \\
\hline Cyclic Prefix $[\mu \mathrm{s}]$ & 7.58 & 8.95 & 22.0 & 26.1 \\
\hline
\end{tabular}

\section{CDACS}

As described in [1], CDACS uses Orthogonal Frequency Division Multiplex (OFDM) in the FL and Single-Carrier Frequency Division Multiple Access (SC-FDMA) in the RL. In this campaign, only the FL waveform is tested. Four different OFDM configurations are used as given in Table 1. Multiple symbol alphabets (4-QAM, 8-PSK, 16QAM, 64-QAM) and Forward Error Correction Codes (Convolutional Code with rates $R_{0} \approx 1 /$ 3and $R_{1} \approx 1 / 2$ ) are used for all configurations in different combinations.
Since the CDACS signal's bandwidth of approximately $1.5 \mathrm{MHz}$ is much smaller than the overall bandwidth available for the experiment, the remaining bandwidth is used by combining the CDACS signal and an altered version of the channel sounding signal. Hence, the CDACS tests and the channel sounding could be run simultaneously. Thus, the available flight-time of the campaign is used more efficiently, since two different measurements are performed at the same time. To allow for a simultaneous channel sounding and CDACS transmission, the generation of the channel sounding signal gets modified at two stages: First, $N_{\text {null }}=196$ sub-carriers around the DC-carrier are set to zero during the generation of the basis signal according to (1). $N_{\text {null }}$ is chosen to guarantee an interference-free coexistence of both signals, hence additional guard bands were added to the CDACS signal bandwidth. The overall bandwidth blocked in the center of the original signal is approximately $4.8 \mathrm{MHz}$.

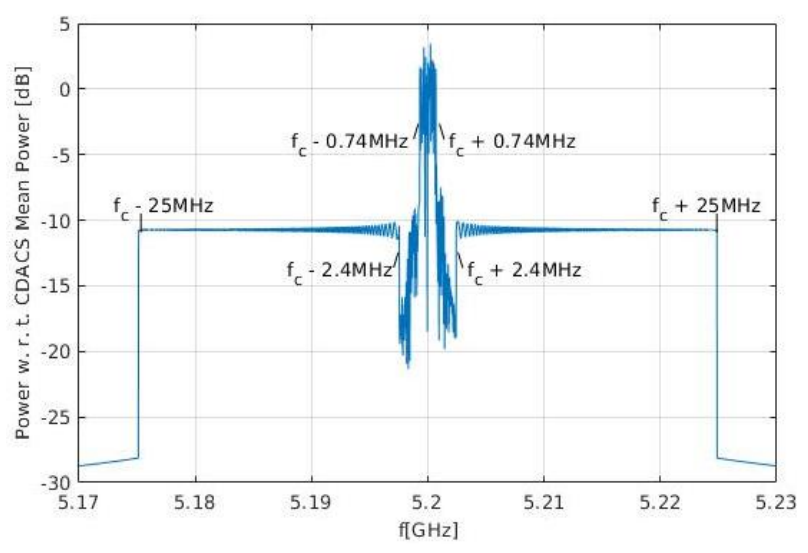

Figure 1. Logarithmic spectrum of the transmitted combined channel sounding signal and the CDACS waveform, $f_{c}=5.2 \mathrm{GHz}$.

Second: The filtering during the iterative PAPR-optimization (2) is extended by an additional rectangular high pass filter matching the conditions given in the previous step.

In a last step, an upsampled version of the pregenerated CDACS signal matching the sample rate of the channel sounding signal and a continuously repeated version of the channel sounding signal are added using a weighted sum. The weight coefficients are representing the power allocation for both signals and are set to $80 \%$ for the CDACS 
signal and $20 \%$ for the channel sounding signal. The spectrum of this combined signal is depicted in Figure 1.

\section{Hardware Setup}

The setup of the campaign consisted of one receiver located onboard the aircraft and one transmitter located on the rooftop of DLR's "Institut für Kommunikation und Navigation" (IKN) building. Hence, data is transmitted from ground to air.
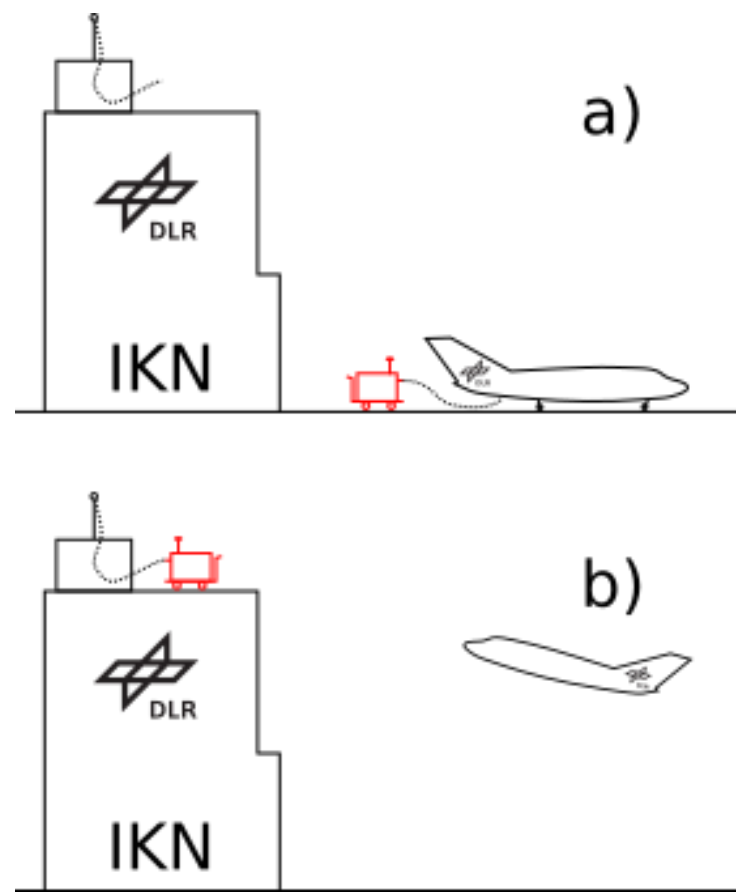

Figure 2. Sketch of the locations of the mobile ground station (red): a) during calibration on the airfield, b) during flight. RF cables are sketched with dashed lines.

Immediately before and after each flight, ground station and air station are directly connected for both synchronizing the Rubidium clocks and performing a calibration measurement. Since the air station is firmly fixed to the aircraft, the ground station is transported to the aircraft waiting on the apron. This motivates the mobile design of the ground station.

The two operation locations of the ground station are depicted in Figure 2. The location during the calibration process on the airfield is sketched in a) while the location during the flight measurements, called transmit spot, is given in b). The entire air station and all parts of the ground station except for the 100W C-band high power amplifier (HPA) have to be kept running between the calibration process and the measurement flights to keep the AWG and the IQ-recorder, among other devices, synchronized to each other. For the ground station, this is realized by using an uninterruptible power supply (UPS) to bridge the time of transportation. The airborne station is running on the power of the auxiliary power unit (APU) of the aircraft, which enables a gapless power supply when the main engines are shut on or off, respectively.

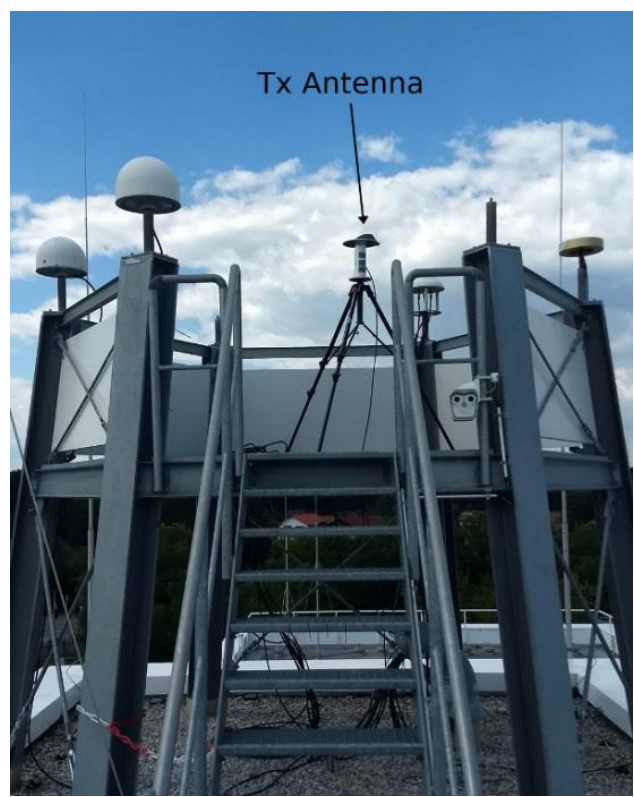

Figure 3. Tx antenna on the rooftop of the IKN building. The transmitting location of the mobile ground station is in the room below the platform.

\section{Transmitter (Ground Station)}

The transmitter station's parts are depicted in Figure 4. While the transmitting antenna and its cable are firmly fixed on the rooftop of the IKN building, see Figure 3, all remaining equipment is located on a mobile platform. The pre-generated signals are loaded into and replayed by the AWG. The output is amplified by the HPA and filtered by a bandpass (BP) filter to reduce spurious emissions and harmonics. The Rubidium (Rb) clock and the Global Navigation Satellite System (GNSS) receiver shape a GPS disciplined oscillator (GDO) 
and keep the AWG synchronized w. r. t. the Global Positioning System (GPS). Besides, GNSS-data (GPS, Galileo, GLONASS, Beidou) is logged to detect possible clock drifts. These drifts must be considered when evaluating the data collected during the campaign. All devices except for the HPA are connected to the UPS to enable an uninterrupted operation when the ground station is moved from the aircraft to its transmit location and vice versa.

During the calibration process a), the BP filter is directly connected to the receiving antenna cable of the aircraft station using a cable comparable to the actual transmit cable ${ }^{4}$ used in the transmit location and some attenuators. For technical reasons, the actual transmit antenna cable had to remain at the transmit location all times. During the channel measurements $b$ ), when the ground station is in its transmit location, the output of the BP filter is connected to the transmitting antenna using the installed antenna cable.

\section{Receiver (Aircraft Station)}

The receiving equipment is split over three common 19" racks onboard the aircraft. Its blocks are depicted in Figure 5. The incoming signal is bandpass-filtered and pre-amplified by a two-staged low noise amplifier (LNA). It is then downconverted to an intermediate frequency of $f_{\mathrm{IF}}=$ $80 \mathrm{MHz}$ before it is fed into a National Instruments PXI system. The PXI system samples the incoming signal at 50MSamples/s, converts it into a baseband signal and stores the samples' in-phase and quadrature components (IQ) to a 12TB RAID. Similar to the ground station, a GNSS-receiver and an Rb-clock building a GDO are used as a time source. Besides, GNSS-, video- and Inertial Measurement Unit (IMU) data is recorded. All recording devices in the receiver use GNSS time information to timestamp the recorded data in a precise manner which is indispensable for postprocessing.

\footnotetext{
${ }^{4}$ Same manufacturer, same product, same batch, (nearly) same length, however, both cables are inspected using a Vector Network Analyzer and differences in attenuation and group delay must be considered during evaluation.
}

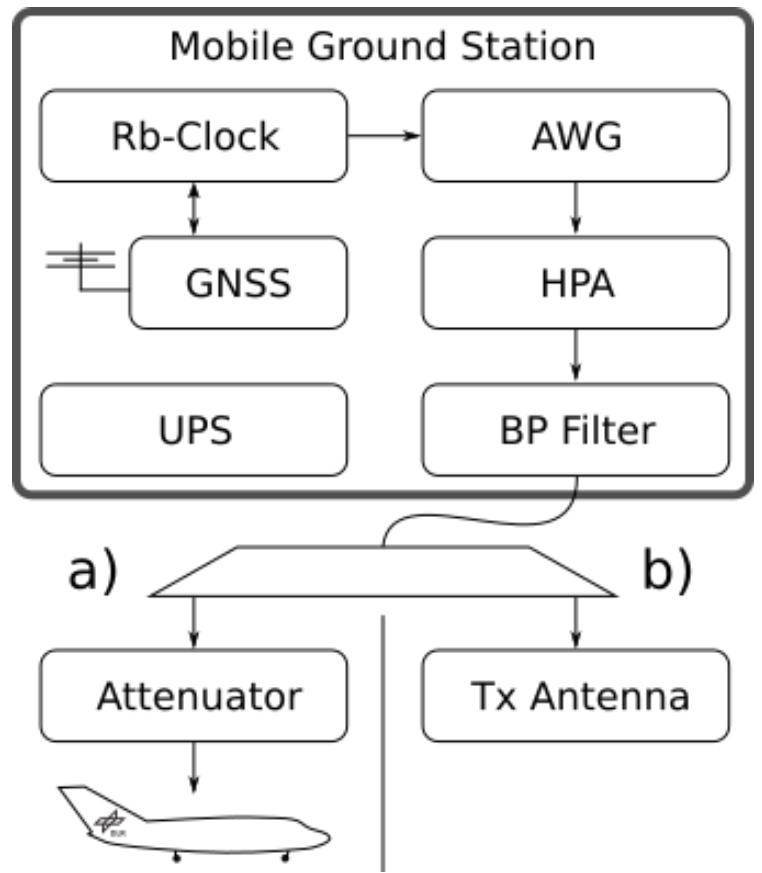

Figure 4. Schematics of the mobile ground station, i.e. the transmitter.

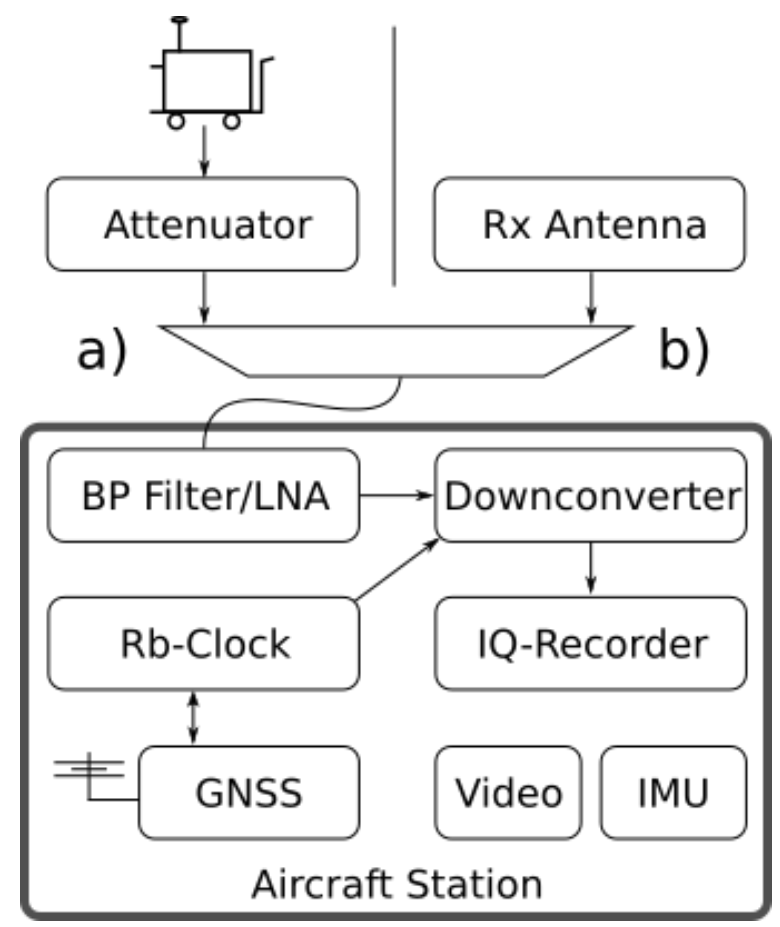

Figure 5. Schematics of the aircraft station, i.e., the receiver. 


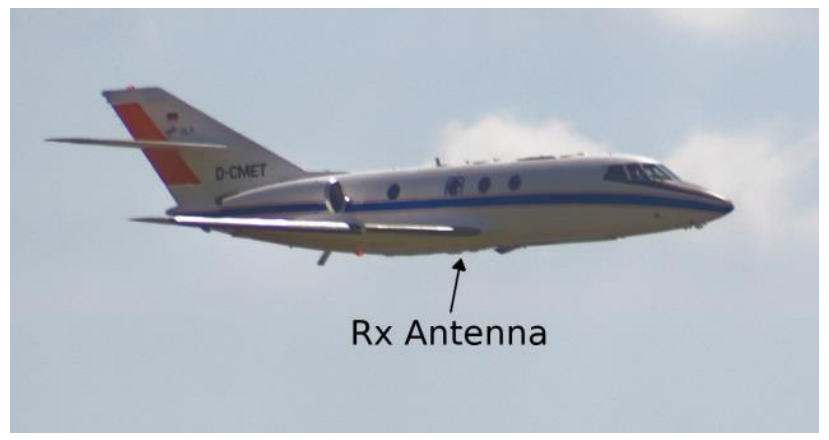

Figure 6. Falcon 20E Aircraft during a missed approach maneauver. The $\mathrm{C}$-Band receiving antenna is mounted at the bottom of the fuselage.

During the calibration process a), the receiving antenna is disconnected and its antenna cable is connected to the ground station using attenuators and another cable as described in the previous subsection. Besides, the Rb-clocks of both the ground station and the aircraft are connected to synchronize their clock signal. During the channel measurement, i.e., during taxi, take-off, en-route and landing, the receiving antenna is connected to the LNA as depicted in b).

\section{Flight Routes and Maneuvers}

The flight campaign consisted of four flights with different flight patterns. All flights have in common that they start and end at the Sonderflughafen Oberpfaffenhofen (EDMO) close to Munich, Germany. The linear distance between the transmitter location and the runway of EDMO is just $\sim 550$ meters. Additionally, several missed approach maneuvers are flown before every landing. The aircraft used was a Dassault Falcon 20E (D-CMET), operated by the German Aerospace Center DLR, see Figure 6. An overview of all flights is given in Table 2; a more detailed description of the flights can be found in the following.

Table 2. List of all Flights of the Campaign

\begin{tabular}{|c|c|c|c|}
\hline$\#$ & Date & Duration & Signal Type \\
\hline 1 & $07 / 09 / 18$ & $3: 20 \mathrm{~h}$ & $\mathrm{CS}$ \\
\hline 2 & $07 / 10 / 18$ & $2: 05 \mathrm{~h}$ & $\mathrm{CS}$ \\
\hline 3 & $07 / 10 / 18$ & $3: 05 \mathrm{~h}$ & $\mathrm{CS}+\mathrm{CDACS}$ \\
\hline 4 & $07 / 12 / 18$ & $1: 30 \mathrm{~h}$ & $\mathrm{CS}$ \\
\hline
\end{tabular}

\section{Flight \#1: Circles around EDMO}

During this flight circles of different diameters are flown at different flight levels (FL); the flight track is depicted in Figure 7. The circles are flown around FL100 and FL370, respectively. The outer circles' radii are around $30 \mathrm{~km}$, the inner circles' radii are around $7 \mathrm{~km}$.

Besides the circles, fly-overs along the northsouth-axis and the east-west axis are conducted.

The turns during the flight and the large "bow" in north-east direction when changing the FL lead to airframe shadowing, where the receiving antenna loses the line of sight (LoS) connection to the transmitter. Thus, large Rx signal power drops are expected.

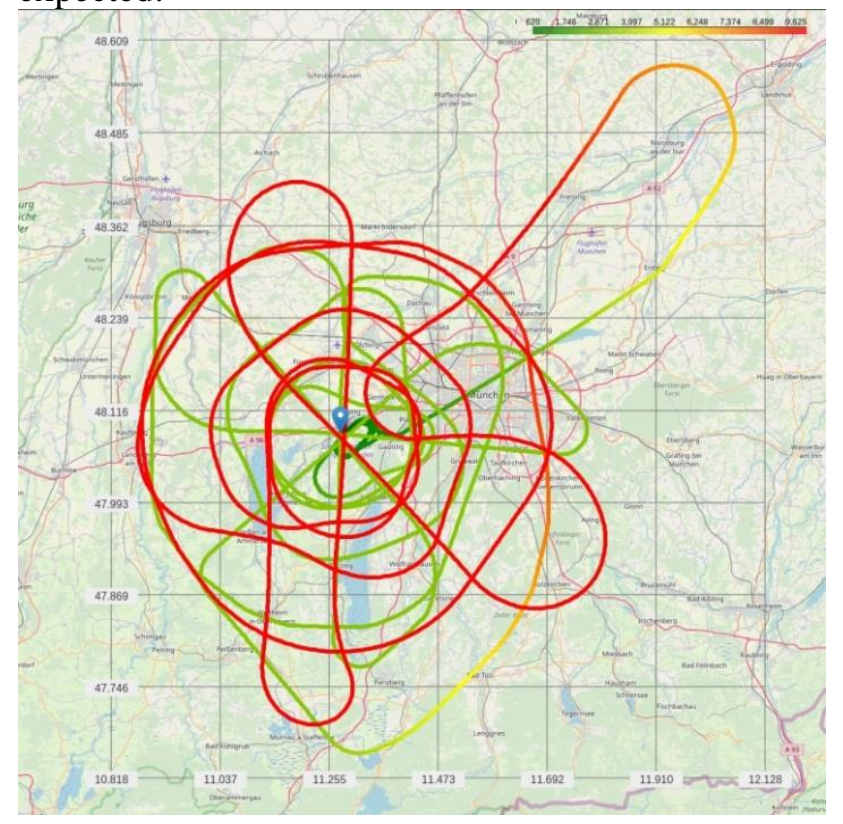

Figure 7. Track of the first flight with colorcoded altitude given in $\mathrm{m}$. The location of the transmitter is depicted by a blue icon.

The circular shape of the flight route segments is motivated by the fact, that the distance to the transmitter is approximately the same for one turn. Thus the FSPL of the LoS component is expected to show just a small variance and observable effects in terms of the received signal strength are likely to have a different origin.

At the end of the flight, missed approaches are flown when approaching the runway of the EDMO airport. The speed during these scenarios is around 
$330-340 \mathrm{~km} / \mathrm{h}$ above ground, the altitude is just around $35-40 \mathrm{~m}$ above the runway.

\section{Flight \#2: Long Distance Flight}

This flight is the only one, where the larger area around the transmitter is left. The long segments along the north-south axis are flown around FL350, the turn was made close to the city of Wolfsburg, roughly $510 \mathrm{~km}$ linear distance away from the transmitter. The last part of the flight time back to EDMO is flown around FL80.

The idea of the long distance flight is to fly beyond the radio horizon i.e., to lose the LoS connection to the transmitter at EDMO. Additionally, a varying receiving power is expected before leaving the radio horizon; not only caused by the increasing free space path loss (FSPL), but by constructive and destructive interference of the LoS signal path with the ground reflection signal path. The observability of this interference effect is expected to be better during a long distant flight heading directly away from or towards to the transmitter, respectively.

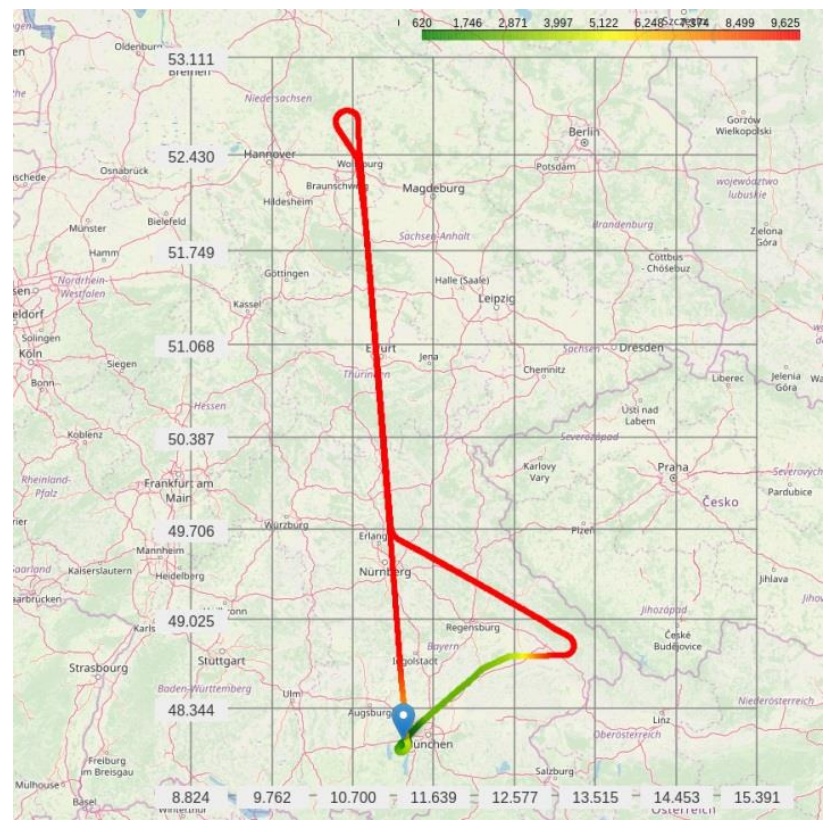

Figure 8. Track of the second flight with colorcoded altitude given in $\mathrm{m}$. The location of the Transmitter is depicted by a blue icon.

\section{Flight \#3: Squares around EDMO with CDACS}

During this flight squares of different sizes are flown at different flight levels (FL). This flight is the only one, where the CDACS signal and the channel sounding signal are transmitted jointly. Both the squares and the fly-overs are flown two times: One time around FL105 and one time around FL210. The squares' edge lengths are approximately $22 \mathrm{~km}$ and approximately $52 \mathrm{~km}$, respectively.

The square shape has the advantage of a straight heading direction. However, the distance to the transmitter is varying together with the Doppler shift while passing by.

\section{Flight \#4: Squares around EDMO}

During this flight squares of different sizes were flown at different flight levels (FL). This flight was performed around FL155 most of the time. The square dimensions are the same as for flight 3; however, the squares are rotated by 45 degree. The rotation of the same shape provides a comparable setup w. r. t. the transmitter but different conditions for the ground reflected wave.

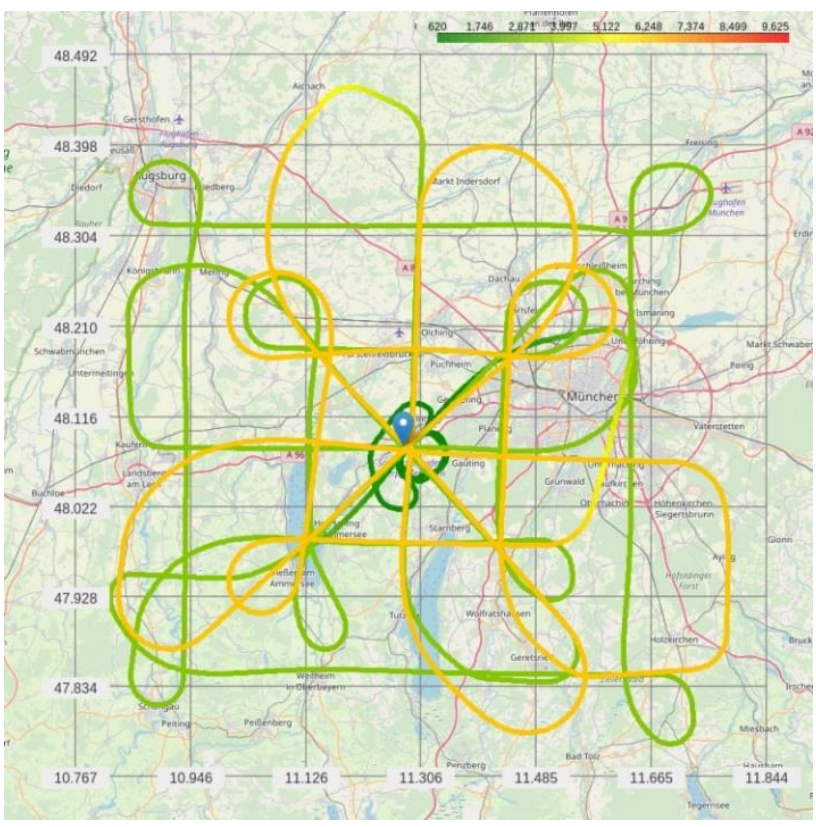

Figure 9. Track of the third flight with colorcoded altitude given in $\mathrm{m}$. The location of the Transmitter is depicted by a blue icon. 


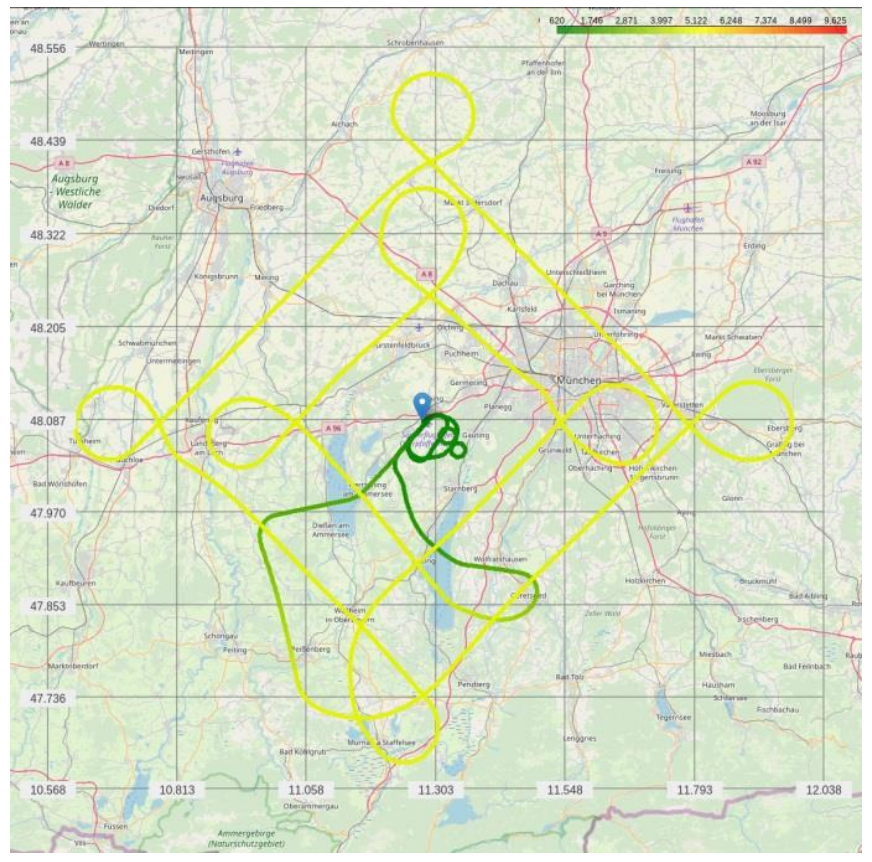

Figure 10. Track of the fourth flight with colorcoded altitude given in $\mathrm{m}$. The location of the Transmitter is depicted by a blue icon.

\section{First Results}

The evaluation of the campaign data is still an ongoing process. However, to give the reader an idea of what kind of results can be expected, we present a preliminary result as an example.

Figure 11 shows the spectrum of the received signal of the combined channel sounding and CDACS waveform during taxi, similar to the transmitted signal given in Figure 1.

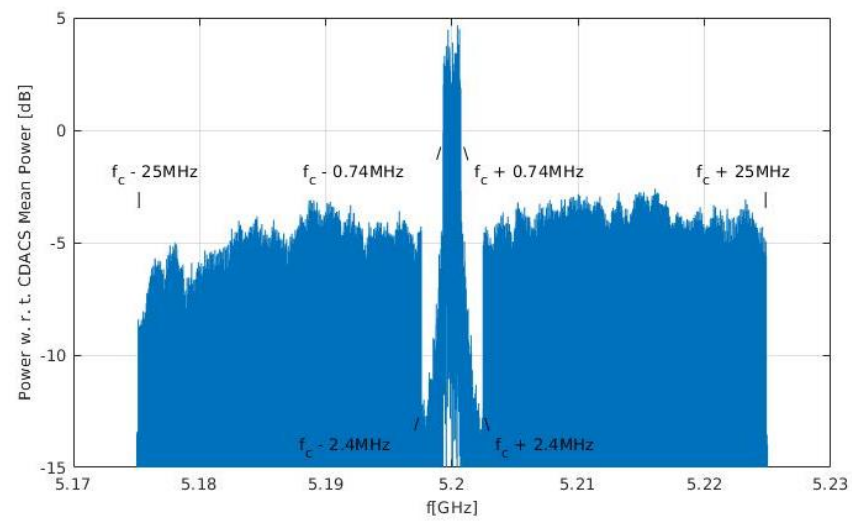

Figure 11. Logarithmic $R x$ spectrum of the combined channel sounding signal and the CDACS waveform during taxi, $f_{c}=5.2 \mathrm{GHz}$.
Both the CDACS signal and the channel sounding signal can be easily perceived and visually identified. Especially when comparing the transmitted and the received spectrum of the channel sounding signal, the impact of the channel on the signal gets evident. The channel shows the behavior of a multipath channel and adds fading effects to the signal.

\section{Conclusion \& Outlook}

This paper gives an overview of DLR's Cband measurement campaign of July 2018. The motivation, the transmitted signals, the hardware setup and the flight routes have been explained.

The evaluation of the collected data is still ongoing. The aim is to use the processed data to create a detailed channel model similar to [4].

\section{References}

D. M. Mielke, "C-Band Digital [1] Aeronautical Communication for Unmanned Aircraft systems," 36th Digital Avionics Systems Conference Proceedings, Sep 2017.

D. M. Mielke, "Frame Structure of the C[2] Band Digital Aeronautical Communications System (CDACS)," 2018 Integrated Communications Navigation and Surveillance (ICNS) Conference, April 2018.

S. Boyd, "Multitone Signals with low crest [3] factor," IEEE Trans. Circuits Syst., pp. 1018$1022,1986$.

N. Schneckenburger, "Reflector [4] Localization for Geomatrical Modeling the AirGround Channel," IEEE Transactions on Vehicular Technology, pp. 7994-8008, Sept. 2018.

\section{Acknowledgements}

The measurement campaign was supported by Nils Mäurer, Lukas Schalk, Dennis Becker, Miguel Bellido, Alexandra Filip, Thomas Gräupl, Michael Schnell, and many others.

\section{Integrated Communications Navigation and Surveillance (ICNS) Conference}

April 9-11, 2019 